\title{
El posicionamiento epistemológico, metodológico y encuadre teórico de una investigación en curso
}

\section{The epistemological position, methodological and theoretical framework of an ongoing research.}

\author{
Mgter. María Cristina R. Alonso ${ }^{1}$
}

Fecha de recepción: agosto 2010

Fecha de aceptación y versión final: marzo 2011

Resumen: Este artículo versa sobre el posicionamiento epistemológico, metodológico y encuadre teórico del cual se partió en la investigación que se está llevando adelante sobre El acompañamiento y sus configuraciones en el ciclo de las prácticas de la futura profesión ${ }^{2}$. Estudio este que tiene una continuidad temática y temporal, pues desde 1999 parte del equipo viene trabajando en esta línea de investigación en diferentes campos de la formación profesional.

Palabras claves: acompañamiento, configuración, ciclo de las prácticas, formación

\begin{abstract}
This paper focuses on the epistemological position, methodological and theoretical framework, from which the research has started and which is being taken forward on the accompaniment and its configurations during the training for the future profession. This study has a thematic and a temporal continuity. Since 1999, a group of researchers has been working in this kind of research in different fields of vocational training.
\end{abstract}

Keywords: accompaniment, configuration, cycle practices, formation.

\footnotetext{
${ }^{1}$ Facultad de Humanidades - UNNE - correo electrónico: macristialonso@ yahoo.com.ar

${ }^{2}$ Se la está llevando adelante con un equipo de investigación: Profesoras Especialistas Zulma Susana Difilipo, Nilda Beatriz Ramírez y Mónica Beatriz Vargas; y con el apoyo técnico de las profesoras Marlene Paola Quintana, Tamara Romina Correa, Cristina Ramona Ramírez y la Lic. Guadalupe Rocío del Valle Leiva. Aprobado por la Secretaria General de Ciencia y Técnica de la Universidad del Nordeste, Proyecto 123/2007 SCyT U.N.N.E. Unidad Ejecutora: Departamento de Ciencias de la Educación. Instituto de Ciencias de la Educación. Facultad de Humanidades. U.N.N.E.
} 


\section{Introducción}

Lo que aquí se expone se refiere al posicionamiento epistemológico y metodológico, así como a las categorías teóricas de las que se partió en la investigación que se está llevando adelante sobre El acompañamiento y sus configuraciones en el ciclo de las prácticas de la futura profesión.

Este estudio tiene una continuidad temática y temporal, pues desde 1999 parte del equipo viene trabajando en una línea investigación que se centra en los modos en que se configura el proceso de acompañamiento durante el ciclo de las prácticas en diferentes campos de la formación profesional. En esta oportunidad se han tomado como casos: el ciclo de las prácticas en una institución de formación docente no universitaria y en la formación de arquitectos en una universidad nacional estatal ${ }^{3}$.

El recorrido transitado proporcionó información relevante y demostrativa, a través de distintos tipos de material empírico, en relación con las problemáticas que acontecen en el ciclo de las prácticas profesionales durante la formación de grado. Entre las problemáticas sustantivas se podrían señalar:

(a) las diferentes concepciones sobre la relación entre la teoría y la práctica que cohabitan durante el ciclo de las prácticas, particularmente en los alumnos ${ }^{4}$;

(b) el modo en que las condiciones institucionales ${ }^{5}$ operan durante el proceso de acompañamiento; cuando tales condiciones obturan la posibilidad de un acompañamiento en sentido clínico, los docentes del ciclo de las prácticas, muchas veces, apelan a ideologías defensivas ${ }^{6}$ contra el sufrimiento que ello produce; sufrimiento no identificado por los que lo padecen ocupados en realizar el esfuerzo por llevar adelante la tarea, al desplegar mecanismos defensivos contra él ${ }^{7}$;

(c) la violencia simbólica con apelación a la culpabilidad, naturalizada en la relación pedagógica, que da cuenta de la institución del poder no analizada e interpretada durante el acompañamiento a los estudiantes en sus procesos de formación ${ }^{8}$;

(d) las representaciones que los alumnos (caso profesorado en Ciencias de la Educación) tienen de su futura profesión docente, se circunscriben a prácticas pedagógicas que, parafraseando a E. Achilli (2000), se localizan en el espacio del aula dentro del sistema educativo formal. Tales representaciones restringen la mirada en

\footnotetext{
${ }^{3}$ En los anteriores estudios se tomó como caso el ciclo de la práctica en la formación de profesores en una institución universitaria estatal.

4 "Obstáculos que afectan la relación teoría-práctica en las prácticas de formación", entre los años 1999-2002 (Alonso, M. C.; Martini, B. A. y Ormaechea, S.)

5 “...dada una situación, todos aquellos aspectos preexistentes al fnómeno que estudiamos, y que establecen con él alguna relación de determinación.” (Fernández, L. 1998: 46)

${ }^{6}$ La ideología defensiva de los oficios tiene como objetivo principal enmascarar, contener y ocultar una ansiedad particularmente grave, es un mecanismo defensivo colectivo y esta dirigida no contra una angustia resultante de conflictos intrapsíquicos de naturaleza mental, sino que está destinada a luchar contra un peligro y un riesgo reales, que al estar dotada de una cierta coherencia realiza adaptaciones relativamente rígidas con la realidad con el riesgo de que aparezcan consecuencias más o menos graves en el plano práctico y concreto. C. Dejours (1992)

7 "Dispositivos de acompañamiento en la formación docente" entre los años 2003-2007 (Alonso, M. C.)

8 "Las relaciones de poder en la relación pedagógica". Vargas, M.; 2006.
}

Revista del Instituto de Investigaciones en Educación. Año 2 - Nº2 - Año 2011 - (5 - 16) ISSN $1853-1393$

Resistencia - Chaco - Argentina 
cuanto al campo de desempeño profesional y produce una cierta vaguedad respecto de los alcances de las competencias profesionales en el campo de las ciencias de la educación ${ }^{9}$.

Las problemáticas mencionadas y su significatividad en relación con el proceso de acompañamiento durante el ciclo de prácticas de la futura profesión, parecen mostrar que tal proceso adquiere peculiares configuraciones o modos particulares de acompañamiento según cómo perciben los involucrados las siguientes dimensiones:

- $\quad$ el tiempo y el espacio de la formación;

- las concepciones sobre la relación teoría - práctica, las que muchas veces se constituyen en teorías implícitas;

- las condiciones institucionales para llevar adelante el proceso de acompañamiento.

El material disponible permitió conjeturar que tales dimensiones tienen una incidencia substancial en los diferentes modos de configuración del proceso de acompañamiento, según sea el posicionamiento de los actores involucrados (docentes y alumnos) en el ciclo de las prácticas de la futura profesión.

La conjetura de base dio origen a la siguiente "interrogación de partida" que actuó a modo de "brujula" durante todo el proceso: ¿cuáles son los modos en que se configura el proceso de acompañamiento durante el ciclo de práctica profesional, en diferentes campos de desempeño, según sea la futura profesión?

\section{El posicionamiento epistemológico y metodológico ${ }^{10}$.}

Tres perspectivas se estimaron decisivas, desde el inicio de esta línea de investigación, en relación con el posicionamiento epistemológico: Thomas S. Kuhn, Fritjof Capra y Edgar Morin. El primero, por cuanto revolucionó con su tesis historicista respecto del fenómeno de la ciencia y con su orientación más sociológica respecto a la noción de paradigma ${ }^{11}$. El segundo, por cuanto generaliza la noción de paradigma científico elaborado por Thomas Kuhn al ámbito de lo social y habla entonces de paradigma social. El tercero, por

\footnotetext{
9 "Las representaciones que los alumnos del quinto nivel de la carrera tienen acerca de lo que es ser un profesor en ciencias de la educación". Vargas, M. B. y Ramírez, N. 2007.

${ }^{10}$ Parte de lo que aquí se expone se extrajo del Trabajo: Alonso, M. C. y Ormaechea S. "La Decisión epistemológica", 2005. Presentado en el Seminario Epistemología y Formación en el marco de la carrera de Maestría en Formación de Formadores (UBA)

${ }^{11}$ La necesidad de pensar en la definición de paradigma de T. Kuhn que se refiere a las ciencias "duras" como la física o la química- y extender su alcance a las ciencias sociales sesga, de suyo, una presuposición esencial: la idea de pseudos ciencia atribuida a las ciencias sociales y, por ende, la debilidad que presentarían sus teorías en cuestiones de confiabilidad, validez y certidumbre. No hay aquí una intención de revivir la larga discusión a que se ha asistido en los últimos treinta años, sin embargo, al retomar la noción de paradigma de F. Capra parece fundamental como punto de partida para demarcar el status científico de las ciencias sociales.
}

Revista del Instituto de Investigaciones en Educación. Año 2 - Nº2 - Año 2011 - (5 - 16) ISSN $1853-1393$

Resistencia - Chaco - Argentina 
cuanto es uno de los que desarrolla el paradigma del pensamiento complejo desde una posición dialógica y culturalmente inscripta ${ }^{12}$.

A partir de estas tres perspectivas, se puede recuperar lo histórico, lo social, lo cultural, lo institucional y lo subjetivo como dimensiones que transversalizan lo real que de este modo pone en evidencia la complejidad ${ }^{13}$ así como cualquier objeto de estudio en el cual las dimensiones aludidas se entrelazan, se interrelacionan e interaccionan en un movimiento recursivo. El modo particular en que se entrelazan esas dimensiones, instala una visión del mundo desde la cual los sujetos conocen, piensan y actúan, y la comunidad se organiza. Todo ello conlleva la no neutralidad de los sujetos y supone un enfoque holístico y un abordaje heurístico, comprensivo, que se opone a la simplicidad y a la linealidad. Por lo tanto, tiene que ver con un modo de conocer, pensar y actuar del investigador en relación con el objeto de estudio y el modo de abordarlo. Es así que se consideró necesario -durante el transcurso de la investigación-: tener en cuenta distintas miradas, elaborar una descripción de las interdependencias entre los mundos simbólico y fenomenológico construidos por los sujetos implicados -docentes y alumnos- y el análisis que hace el investigador para comprender e interpretar el objeto de estudio sin hacer reduccionismos, simplificaciones ni explicaciones asentadas en un esquema racionalizador.

Entonces, el análisis de las configuraciones sobre los modos de acompañamiento durante el ciclo de prácticas de la futura profesión, no puede apuntar a descomponer o desmontar ${ }^{14}$ como en el caso de pensar al objeto como complicado sino de aprehenderlas globalmente en sus aspectos heterogéneos a partir de procesos de familiarización, de escucha clínica para obtener los datos. Es a partir de esos procesos que se decidió comprender ${ }^{15}$ e interpretar ${ }^{16}{ }^{17}$ esa realidad en estudio.

Este encuadramiento, implica que:

\footnotetext{
${ }^{12}$ Estos tres representantes son algunos de los que dieron nombre al cambio en las formas de mirar y hacer, sin embargo no podemos dejar de mencionar aquellos que hace un par de décadas fueron marcando esos cambios que se volcaron en diseños y materializaciones tales como: el pensamiento sistémico de L. von Bertalanffy; el constructivismo de J. Piaget; el construccionismo social de P. Berger, de T. Luckmann, de B. Pearce; el paradigma holográfico de Pribram; las concepciones enactivas de F. G. Varela; la nueva alianza con I. Prigogine e I. Stengers; el paradigma del caos de Gleick; la autopoiesis (patrón de vida, el hacerse a sí mismo) de H. R. Maturana; el paradigma indiciario de Guinsburg; el pensamiento complejo de H. Atlan, C. Castoriadis, Vigotsky, J. Brunner (las voces personales); el aporte de la lingüística de Lakoff y Johnson.

${ }^{13}$ Etimológicamente, los términos complejo y complejidad derivan del latín complexus que significa dar vueltas, entrelazar, entretejer, y también abarcar, contener. Actualmente, el sentido predominante es lo que contiene, abarca y reúne varios elementos distintos, incluso heterogéneos. En la complejidad, el análisis no apunta a descomponer, a desmontar o a deconstruir, sino a comprender más que explicar. (Ardoino J. 1998).

${ }^{14}$ Tal como lo plantea J. Ardoino (1998).

${ }^{15}$ Primera aproximación al objeto. El investigador toma contacto con esa realidad y la transforma en "dato" a través de un proceso de comprensión. Se trata de pasar de "lo real" a su construcción como "dato". En: Souto, M. (1996).

16 "Segunda aproximación. ...pasamos de la comprensión a la interpretación en tanto se recurre a teorías como herramientas heurísticas que permiten una profundización en la significación de los datos. (...) Nuevo sentido que se apoya en el anterior, pero que va más allá iluminando el escenario (...) desde nuevas ópticas. Se pone en juego la elucidación teórica.” En: Souto, M. (1996) Op. Cit.

${ }^{17}$ En el sentido de descubrir significados.
}

Revista del Instituto de Investigaciones en Educación. Año 2 - Nº2 - Año 2011 - (5 - 16) ISSN $1853-1393$

Resistencia - Chaco - Argentina 
los sucesivos acercamientos que se puedan alcanzar en una investigación no son verdades absolutas, sino que cada uno de ellos encierra a la vez lo cierto y lo incierto. La búsqueda de fundamentos ciertos y seguros para el conocimiento en donde se perpetua el retorno a la certidumbre por la verificación en demanda de una Verdad fundadora, tal como pretendió el positivismo lógico, ya no es posible; todo lo cual entraña una renuncia a lo acabado, lo absoluto, lo exhaustivo, a un conocimiento con fundamento indubitable (Morin, E.; 1977).

el investigador no puede separarse del proceso de investigación que lleva adelante (el investigador como sujeto cultural y socialmente situado, es decir, como subjetividad que se fue configurando en un espacio y un momento histórico social específico); así como tampoco el objeto de conocimiento puede aislarse de su entorno institucional y socio-histórico ni del investigador, como si fueran "objetos inertes, inmóviles, inorganizados, cuerpos siempre enmudecidos por leyes exteriores" (Morin, E. 1977: 412).

El investigador es un sujeto en dos lugares: sujeto de la consciencia y sujeto del inconsciente (Green, A. 1991:179) lo que nos está hablando de la percusión subjetiva del objeto de conocimiento, de la otra polaridad subjetiva. Lo que implica no desinvestir al sujeto, no desubjetivarlo en nombre de la objetivación como hacía el paradigma del positivismo lógico o el del conocimiento ingenuo. Por el contrario, lo importante es hacer viable un conocimiento objetivable de la subjetividad, de esta manera se abandona la ilusión de que aquél sea alguien externo a lo que se conoce y se asuma incluido. Cuando conocemos estamos aceptando que nos aproximamos y a la vez nos entreveramos con lo incógnito y heterogéneo del objeto a conocer y del sujeto que conoce; es así que ya no es posible pensar en la imparcialidad del investigador; el sujeto que conoce no puede separarse del objeto a modo de un investigador neutral y externo así como el objeto no puede concebirse totalmente independiente del investigador y ser reducible a elementos simples (deconstruido y reconstruido en forma idéntica) y susceptible de transparencia.

Lo expuesto hasta el momento encierra dos supuestos epistemológicos que orientaron la decisión metodológica. El primero de ellos se relaciona con la naturaleza de la realidad que se investiga como algo complejo que se construye, y para cuya interpretación y comprensión se requiere de múltiples miradas, sin aislarla del campo del cual surge y reconocer, además, que no puede ser abarcado en su totalidad ni que aquello que se comprende de esa realidad es la verdad absoluta. El segundo, alude a la relación intersubjetiva entre el investigador y lo investigado en la cual el fenómeno de la implicación está siempre presente. En otras palabras, resulta ineluctable llevar adelante el proceso de análisis del fenómeno de la implicación de los investigadores. Por eso la reflexibilidad de la que nos habla E. Morin, por eso la autoreflexión de la que nos habla J. Ardoino, en definitiva: por eso la necesidad de considerar el fenómeno de la implicación que tiene que ver con el orden de lo replegado sobre sí mismo y que debe ser comprendida desde una aproximación clínica.

De estos dos supuestos se derivan formas particulares de conocer la realidad a investigar conducidas por una lógica cualitativa que se caracteriza por construir teoría a partir de los datos, en el sentido de significados dados tanto por los que participan directamente en la realidad estudiada como por el/los investigador/res, es decir, comprender el fenómeno estudiado desde la perspectiva de los sujetos implicados a partir de la observación y la escucha atenta.

\footnotetext{
Revista del Instituto de Investigaciones en Educación. Año 2 - N $\mathrm{N}^{\circ} 2$ - Año 2011 - (5 - 16) ISSN $1853-1393$

Resistencia - Chaco - Argentina
} 
Posicionados así, y teniendo en cuenta las problemáticas emergentes de las investigaciones realizadas por parte del equipo (mencionadas más arriba), se adoptó el estudio de casos en profundidad desde un enfoque clínico ${ }^{18}$, con conciencia de que la teoría de base que se elabora no puede ser generalizada; no obstante esto, sí podría ser de interés para quienes están implicados, de una u otra manera, en la cotidiana tarea de llevar adelante experiencias situadas en el ciclo de las prácticas durante la formación de grado.

La selección de los casos obedeció fundamentalmente a su interés intrínseco (Stake, R. E. 1999: 16). El del ciclo de las prácticas en la formación docentes en una institución de formación superior no universitaria, se deriva de interrogantes surgidos en la investigación 2003-2007 (Alonso, M. C.) cuyo campo de indagación estaba ubicado en una institución superior universitaria de formación docente, de tal manera que el caso en cuestión se situa como paradigmático respecto del anterior (institución universitaria e institución no universitaria). En relación con el ciclo de las prácticas en la formación de arquitectos, responde al interés de abarcar las carreras que se ofrecen en las cuatro facultades asentadas en una de las sede de la universidad. ${ }^{19}$

\section{Relevamiento y análisis de la información.}

En primer lugar, se llevó adelante una recuperación de las conjeturas iniciales así como de las hipótesis y problemáticas que como resultados aparecían en las investigaciones anteriormente mencionadas. En esta recuperación se evidenciaba claramente, desde los primeros estudios (1999), la preocupación por querer adentrarse en la comprensión del fenómeno del acompañamiento durante el ciclo de las prácticas en la formación de grado. Esta preocupación es posible pensarla -parafraseando a N. Yentel (2009)- a partir del tipo de vínculo que construimos con el objeto a investigar. Esta autora nos dice: "El objeto de investigación, entonces, es siempre un objeto significado y, como tal -me atrevo a decirdeseado y rechazado al mismo tiempo; una cuestión del cual quisiéramos alejarnos pero que nos atrae y desafía simultáneamente y, de allí su potencial." (Yentel, N; 2009. p. 48). Llevar adelante la historia natural de la investigación y su análisis, entonces, es una condición esencial dentro de este encuadre, es uno de los caminos para abordar el análisis de la implicación y el proceso de validación.

En segundo lugar, para la recolección de los datos primarios, se tomaron dos fuentes de información: la documental y la testimonial. Esta recolección se la inició previo acuerdo con las instituciones y los docentes a ser entrevistados y observados a fin de, por un lado, mantener cierta libertad para desplazarse dentro de la institución, consultar documentación y establecer comunicación con sus miembros y, por el otro, reducir la percepción por parte de los actores institucionales, de "sentirse espiados" (Acevedo, M. J. 2000). Se partió del supuesto de que el adentrarse en terreno para la recolección de la

\footnotetext{
${ }^{18}$ Este enfoque encierra una posición frente al conocimiento y a la realidad que se investiga e implica un estudio en profundidad de un sujeto, de un grupo, de un caso, de un tema (Souto, M Algunas notas sobre la investigación clínica en educación y formación. Ponencia en: I Jornadas Nacionales de Investigación. Fac. de Educ. Elemental y Especial UNCuyo. 2007.

${ }^{19}$ Por Resolución de 1080/09 CS - UNNE- C y T.- se aprueba el nuevo proyecto en el que se incluyen como caso: el ciclo de las prácticas de las carreras de Ingeniería y Ciencias Económicas.
}

Revista del Instituto de Investigaciones en Educación. Año 2 - Nº2 - Año 2011 - (5 - 16) ISSN $1853-1393$

Resistencia - Chaco - Argentina 
información, en tanto intervención de investigación, implica situaciones que pueden ser provocadoras de cambios en las formas de percibir y actuar, en tanto ofrece la posibilidad de repensar las cuestiones tratadas.

La fuente documental si bien fue pensada, primero, desde la definición y clasificación de MacDonald y Tipton (1993:188, citado por M. S. Valles, 1997): "cosas que podemos leer y que se refieren a algún aspecto del mundo social. (...) esto incluye aquellas cosas hechas con la intención de registrar el mundo social -informes oficiales, por ejemplo- pero también los registros privados y personales"; luego, se esclareció que tal fuente también encierra el posicionamiento de aquellos que en diferentes momentos tuvieron lo medios para asentar de manera escrita normativas y/o sucesos que pueden estar teñidos de intereses particulares (Guha, 1999; citado por E. Rockwell, 2009).

Los documentos seleccionados de los casos en estudio, representan el marco referencial y regulatorio que intervienen influyendo, de una u otra manera, en la percepción de los sujetos involucrados (docentes y alumnos) sobre el proceso de acompañamiento y, como consecuencia en el modo en que se configura durante el ciclo de prácticas de la futura profesión. Los documentos analizados son los diseños curriculares de las carreras involucradas, los programas de los espacios curriculares pertenecientes al ciclo de las prácticas y normativas específicas al mencionado ciclo. Además, y de acuerdo con lo explicitado respecto del posicionamiento epistemológico y metodológico, el diario de campo de campo de los investigadores.

La fuente testimonial incluye entrevistas (abiertas individuales y grupales) y observaciones. Las entrevistas en tanto proceso comunicacional, encierran en el plano del contenido un sistema de significación sobre los acontecimientos vividos por el/los entrevistado/s (Delgado, J. M. y Gutiérrez, J.; 1999); proporcionan relatos cruzados de las personas de un mismo entorna sobre el objeto que se estudia o -parafraseando a M. J. Acevedo; 1999- ofrecen la posibilidad de escuchar varias voces que en conjunto y a través de los relatos, explicaciones y comentarios de los entrevistados se rescatan y ahondan las dimensiones simbólica e imaginaria del fenómeno que se investiga. Las observaciones fueron directas en situación natural con registros sin categorías previas de tal manera de "ser testigo de los comportamientos sociales de individuos y grupos en los espacios mismos en que realizan sus actividades, sin modificar el desarrollo habitual de los mismos" (Henri Peretz, 1998 citado en Acevedo, M. J. 2000).

En ambos casos de estudio, las entrevistas abiertas individuales se realizaron a los profesores -según las diferentes posiciones ${ }^{20}$ : titular, adjunto, jefe de trabajos prácticos, auxiliares, adscriptos- que integran el equipo docente de los espacios curriculares, y las entrevistas grupales a los alumnos cursantes. Ambos tipos de entrevistas se basaron en áreas de indagación previamente establecidas a partir del problema planteado y son las que guiaron el escudriñamiento en la situación de entrevista. Se utilizó el registro de forma escrita en simultáneo con grabación magnetofónica. La duración promedio de cada entrevista fue de una hora y media. Se las organizó en dos tiempos. En el primero, se solicitó a los entrevistados que hablaran libremente a partir de las grandes áreas

\footnotetext{
${ }^{20}$ La posición es la ubicación del sujeto en la trama relacional de los sistemas de poder. Esa trama relacional está enmarcada en el conjunto de normas y reglas que señalan lo permitido y lo prohibido en un espacio social determinado. (Fernández, L.; 1994).
} 
temáticas. Luego de un descanso, se entregó al/los entrevistado/s una copia escrita con las áreas para que profundizaran o agregaran lo que les pareciera necesario.

Las dimensiones de análisis trabajadas en los anteriores casos investigados, se tomaron como punto de partida para la definición de las áreas de indagación, por cuanto habían resultado altamente significativas para adentrarse en el objeto en estudio. Ellas son: el diseño del proceso de acompañamiento, es decir, los propósitos y contenidos formales explicitados, las acciones y los materiales -en tanto recursos- que prevé, la vinculación con el ciclo de prácticas en general previsto en el currículo de la formación docente; el desarrollo del proceso de acompañamiento, es decir, el modo de intervención tanto de/los docente/s a cargo de los espacios curriculares del ciclo de las prácticas como de los de los sujetos involucrados en el futuro campo de desempeño, los propósitos y contenidos que efectivamente se desarrollan, las acciones que realizan y cómo se las secuencia, qué materiales trabajan y cómo los trabajan, las teorías sobre el modo de acompañamiento que sustentan y sus fundamentos, los materiales que produce el alumno; la dinámica del proceso de acompañamiento, es decir, cómo se desarrollan los hechos, su acontecer, cuáles son los momentos más significativos del proceso de acompañamiento, las tensiones y conflictos que se producen, cómo se plantean y resuelven las dificultades, las normas explícitas o implícitas que operan en esa dinámica, cómo es considerada la variable personal y grupal, las características que presentan las relaciones interpersonales y las relaciones vinculares, cómo se produce y opera la contención y el control emocional; la experiencia de "acompañar y ser acompañado", es decir, cómo es vivido el proceso de desarrollo en el dispositivo de acompañamiento; cómo se percibe, se piensa, se vive el pasaje de la institución de formación inicial al futuro campo de desempeño profesional; cuál es el sentido y el significado que los docentes y los alumnos involucrados, le dan al proceso de acompañamiento.

En uno de los casos $^{21}$ se incluyó un grupo de reflexión (un encuentro mensual: 10 en total) con todos los profesores del ciclo de las prácticas de los distintos profesorados. Este instrumento, además de servir para la recolección de información y como efecto del encuadre de investigación adoptado, operó a su vez como instancias de acompañamiento a los docentes en su proceso de formación durante la experiencia. Llevar adelante el grupo de reflexión en este contexto, representó un desafío por cuanto requería respetar los tiempos y los requerimientos del encuadre metodológico previsto en el diseño y, al mismo tiempo, atender a los tiempos y necesidades planteadas por la "situación de formación" seleccionada como contextos privilegiado de investigación.

Las observaciones se hicieron sobre los distintos espacios de la institución, unos, relacionados con las actividades de enseñanza (aulas, laboratorios en donde se lleva adelante el proceso de acompañamiento a los alumnos en los días estipulados formalmente para ello), otros, con las actividades de conducción así como los relacionados con el ir y venir de la vida cotidiana (pasillos, patio); espacios que a pesar de ser comunes a otras instituciones de formación, presentan formas singulares de mostrarse.

A medida que se fue recogiendo la información se extrajeron en primer lugar, categorías temáticas para luego intensificar el descubrimiento de sentidos y significados (tratando de

${ }^{21}$ Intitución de formación docente superior no universitaria.

Revista del Instituto de Investigaciones en Educación. Año 2 - No2 - Año 2011 - (5 - 16) ISSN $1853-1393$

Resistencia - Chaco - Argentina 
centrarse en la comprensión de la perspectiva en la cual se ubicaban los sujetos), todo ello fundado en el contexto del caso. Considerando que la construcción de significados dan cuenta de interpretaciones sobre datos basados en la realidad, aquellos constituyen un medio poderoso para comprender el mundo que "está allí". Es así que el primer nivel de organización de la información para convertirla en dato -tanto de lo escuchado como de lo observado-, fue la elaboración de categoría temáticas que dio lugar a un relato narrativo descriptivo y a un primer reconocimiento sobre aquellos aspectos que configuran el modo de acompañamiento en cada caso en estudio. Una segunda vuelta al caso, se realizó a partir de un análisis comparativo entre las categorías temáticas de las diferentes fuentes -testimoniales y documentales- a fin de elabor las líneas interpretativas. Esto último posibilitó la contrastación de distintos puntos de vista desde el lugar de donde se encara (tanto desde las diferentes fuentes como desde los investigadores del equipo).

\section{Categorías teóricas: un punto de partida}

El equipo de investigación asumió que "el investigador se aproxima al problema con un caudal de conocimientos de resultados de investigaciones anteriores y con un acervo conceptual de teorías que lo ayudan a delimitar su temática. Estos elementos le permiten al investigador generar hipótesis de trabajo, que no son una relación entre variables operacionalizadas sino una interrelación entre conceptos amplios a redefinir en el propio trabajo cualitativo" (Gallard, M. A., 1992). Desde esta visión, los marcos teóricos operan como analizadores y como fuente para la organización de las categorías interpretativas. Éstas, a su vez, actúan como punto de partida para interpelar la teoría, lo cual exige replantearse (ajustar, precisar, ampliar, desechar, crear) conceptos, procesos, interpretaciones.

A partir de esta lógica, se dilucidaron las categorías teóricas que encierra la interrogación al problema: configuración, acompañamiento, ciclo de las prácticas, formación. Con respecto a la noción de configuración, algunas de las características dadas por Norbert Elías fueron particularmente potentes para su clarificación; así como la propuesta de J. Ardoino para la noción de acompañamiento. En relación con las nociones de ciclo de prácticas y de formación, se recuperaron las que el equipo venía sosteniendo desde las primeras investigaciones que, si bien, en el devenir sufrieron algunas modificaciones no perdieron su significatividad general primera.

La noción de configuración es entendida como el modo singular en el que se disponen las partes que componen una situación y le dan una peculiar forma y propiedades, en un "aquí y ahora"; ese modo singular está sostenido por las relaciones de interdependencia y procesos de integración entre los fenómenos y experiencias de los sujetos Todo ello afectado por la transversalización de lo institucional, lo social y lo histórico. La interdependencia y la integración mantienen una conexión dinámica que conforma un tejido; tal dinámica sólo puede entenderse a partir de la configuración de la cual surge sin dejar de considerar la contingencia. La configuración enfatiza, desde N. Elías, dos niveles de observación diferentes pero inseparables: los sujetos y la red de interdependencia en la que están inscriptos de manera cambiante (Devin, G.; 2005).

La noción de acompañamiento, contiene al tiempo y al espacio. El tiempo como tiempo experimentado de manera subjetiva e íntersubjetiva. El tiempo como temporalidad, como

Revista del Instituto de Investigaciones en Educación. Año 2 - $\mathrm{N}^{\circ} 2$ - Año 2011 - (5 - 16) ISSN $1853-1393$

Resistencia - Chaco - Argentina 
vivencia en la experiencia conciente e inconciente que remite al dilema de la mismidad y de la otredad en el que el sujeto se va haciendo otro por la interferencia de ese otro y por el efecto de la alteración de la reiteración, el cambio, etc.. (Henri Lefebvre, 1992). Inseparable de la temporalidad, el espacio psíquico y social en tanto ámbito para articular lo antiguo y lo nuevo y dar curso a la generatividad, es decir, a la productividad y creatividad, por lo tanto a la posibilidad de generar nuevos productos e ideas, incluido un tipo de autogeneración que tiene que ver con un mayor desarrollo de la identidad. (Erikson, E., 1985). Desde estas concepciones sobre tiempo y espacio que dan lugar al desarrollo de la "alteridad", el acompañamiento permitiría y apuntalaría la "evolución de las relaciones intersubjetivas y, por ello, a una reinterrogación de opiniones, creencias, representaciones, actitudes que expresan los sistemas de valores en juego" (Ardoino, J.; 1997: 86) para el reconocimiento de un saber sobre sí mismo y sobre la situación de trabajo objeto de la práctica. Es decir, el juego implicación-distanciación en sujetos involucrados en prácticas sociales se constituye en eje del análisis con la presencia de un tercero que desde el lugar del otro como acompañante, facilita las reinterrogaciones.

La expresión ciclo de las prácticas de la futura profesión, hace referencia al período por el cual el estudiante atraviesa diferentes experiencias en relación con las prácticas de su futura profesión. Son prácticas formalmente organizadas en los currículos de formación, destinadas, según sus propósitos formales explícitos, a posibilitar que los estudiantes hagan una experiencia en el futuro campo de desempeño, acompañados por el/los docentes de la institución formadora. Además, constituyen una instancia privilegiada de socialización profesional en el que las experiencias ofrecen la posibilidad de incorporar un conjunto de "saberes básicos" que constituyen herramientas centrales del ejercicio profesional e involucran también, la internalización de usos y costumbres derivadas de las "culturas de trabajo" propias de los contextos de desempeño (Andreozzi, M., 1996)

La formación es entendida como la posibilidad de analizar y reflexionar todos los aspectos que se entrecruzan en una situación de práctica; de problematizar los fenómenos que acontecen en la práctica, tornarlos inteligibles por medio de la observación, la escucha y la reflexión. La reflexión sobre lo observado y escuchado tanto de los fenómenos que se dan a nivel externo como de los movimientos internos, es una vía de acceso a los procesos de transformación del sujeto en situación de práctica de una futura profesión (Alonso, M. C.; 2007).

Las condiciones institucionales para llevar adelante tal proceso de acompañamiento, se refieren - siguiendo a L. Fernández, 1998- a aquellos aspectos de la vida institucional que preexisten al fenomeno en estudio y que establecen con él alguna relación de determinación; "condiciones que son recibidas, interpretadas, valoradas y respondidas de formas, muchas veces, diametralmente opuestas, en establecimientos diferentes"(Fernández, L.; 2006:53). Las condiciones instituciones son relevantes, entonces, cuando de algún modo afectan a los sujetos en sus prácticas sociales por los sentidos que le atribuyen a aquellas, influyendo sobre el sentido que le dan a sus prácticas.

Estas categorías teóricas son las que de alguna u otra manera actuaron como esquemas interpretativosa durante la investigación, particularmente a la hora de pensar y re-pensar las hipótesis.

Revista del Instituto de Investigaciones en Educación. Año 2 - $\mathrm{N}^{\circ} 2$ - Año 2011 - (5 - 16) ISSN $1853-1393$

Resistencia - Chaco - Argentina 
Por último, se podría decir que el camino recorrido se asentó en considerar la investigación -tal como la define Achili, E. 2000:20-: como "el proceso por el cual se construyen conocimientos acerca de alguna problemática de un modo sistemático y riguroso". Sistemático en tanto se basa en criterios y reglas lo suficientemente flexibles que permiten definir las condiciones en que se producen determinados conocimientos y, rigurosos, en tanto se busca continuadamente la coherencia de las acciones respecto del problema y las resoluciones metodológicas construidas. Todo ello, bajo el supuesto de que toda intervención de investigación social de índole cualitativa, favorece o produce cambios en los sujetos implicados.

\section{Bibliografía}

Achilli Elena Libia.(2000) Investigación y Formación docente. Laborde editor. Rosario. Argentina.

Andreozzi, Marcela. (1996). "El impacto formativo de la práctica. Avances de investigación sobre el papel de las prácticas de formación en el proceso de socialización profesional." En Revista del Instituto de Investigaciones en Ciencias de la Educación. Año V, Nro.9.

Alonso, María Cristina (2006) «Dispositivos de Acompañamiento ». En: Revista Nordeste. Serie Investigaciones y Ensayos. ISSN 0328-5995 № 25.

Alonso, María Cristina. (2007) Modos de acompañamiento en el ciclo de prácticas de la enseñanza en la formación docente desde la percepción de docentes y alumnos involucrados, un caso particular. Tesis de Maestría. Inédito.

Alonso, María Cristina; Martini, Beatriz Amalia y Ormaechea, Silvia. (2003) "La relación teoría- práctica: los obstáculos que la afectan”. En: Revista Nordeste. Serie Investigaciones y Ensayos. № 15:1-18. ISSN 0328-5995. Resistencia (Chaco).

Ardoino, Jacques. (1997) Complejidad y Formación. Pensar la Educación desde una mirada epistemológica. Bs. As. Ediciones Novedades Educativas. Universidad de Buenos Aires.

Beatriz Sayago, Zoraida y Chacón, María Auxiliadora. "Las prácticas profesionales en la formación docente: hacia un nuevo diario de ruta." Educere, [online] mar. 2006. vol. 10, $\mathrm{n}^{\circ}$ 32, pp 55-66. Disponible en la World Wide Web: http://www.scielo.org.ve/scielo.php?script=sci arttext\&pid=S131649102006000100008\&lng=es\&nrm=iso. [Consulta: marzo 2008].

Devin, Guillaume. "Norbert Elías y el análisis de las relaciones internacionales." Colombia Internacional. Colombia. № 62 jul. - dic. 2005(pp. 174-197)

Erikson, Erik. El ciclo vital completado. Bs. As. Paidós, 1985.

Fernández, Lidia. El análisis de lo institucional en la escuela. Un aporte a la formación autogestionaria para el uso de los enfoques institucionales. Notas Teóricas. Bs. As. Paidós 1998.

(2006) "Espacios institucionalizados de la educación. Algunos componentes nucleares en la identidad institucional y sus consecuencias para el

Revista del Instituto de Investigaciones en Educación. Año 2 - $\mathrm{N}^{\circ} 2$ - Año 2011 - (5 - 16) ISSN $1853-1393$

Resistencia - Chaco - Argentina 
análisis", en Landesmann, Monique (coordinadora). Instituciones educativas. Instituyendo disciplinas e identidades. Méjico, Casa Juan Pablo.

Lefebvre, Henri. "El tiempo denegado en (y por) la escuela". En Actas del Coloquio de la AFIRSE, Lyon, mayo de 1992. Trad. Viviana Rosa Acevedo para uso interno de la cátedra Didáctica II - Facultad de Humanidades de la UNNE-.

Gallard, María Antonia. "La integración de métodos y la metodología cualitativa. Una reflexión desde la práctica de la investigación (1992). Disponible en página Web: http://www.cinterfor.org.uy/public/spanish/region/ampro/cinterfor/publ/arte/gallart/pdf/ca p8.pdf. [Consulta: marzo 2008].

Salamacchia, Homero R. Calón, Héctor y Rodríguez, Javier (1983): "Historias de vida y movimientos sociales:propuestas para el uso de la técnica." Revista Iztapalapa N`9, 321-338. Revista de Ccias. Soc y Humanidades. Universidad Autónoma Metropolitana Unidad Iztapalapa. Méjico. http://148.206.53.230/revistasuam/iztapalapa/index.php. [Consulta:marzo 2009].

Taylor, S. J. y Bogdan, R. Introducción a los métodos cualitativos de investigación. Bs. As., Paidós. 1984 